\title{
Schlussbetrachtung: vom Handel zur Hilfe
}

„Die Schweiz wird in Zukunft auf gewisse Privilegien verzichten müssen“, forderte die „Erklärung von Bern“ 1968. Die Aktivistinnen und Aktivisten im Umfeld der Bewegung erkannten, dass die Dritte Welt eine Anwaltschaft in den Industrieländern brauchte. Dies war das Ziel der Überführung der sozialen Bewegung, die sich im Zusammenhang mit dem Manifest von 1968 konstituiert hatte, in eine ständige Organisation. So entstand die heute noch aktive, professionelle schweizerische Entwicklungs-NGO EvB (seit 2016 Public Eye). Ihre Genese steht jedoch exemplarisch für eine Entwicklung, die wir auch in anderen europäischen Ländern, insbesondere in Grossbritannien und in den Niederlanden, beobachten können. Überall in Westeuropa formierten sich in den Jahren vor 1968 soziale Bewegungen, welche die Umsetzung der UNCTAD-Vorgaben forderten. Überall in der westeuropäischen Dritte-Welt-Bewegung gingen die ursprünglichen Forderungen der UNCTAD nach Marktzugang und Modernisierung verloren und machten einer Duldung, wenn nicht sogar Unterstützung des europäischen Neoprotektionismus, insbesondere im Agrarbereich, Platz.

Wie kam die paradoxe Situation zustande, dass wir heute Hilfe leisten statt Handel treiben? Wie war es möglich, dass genau das Gegenteil eintrat von dem, was die UNCTAD 1964 und die EvB 1968 gefordert hatten? Wie konnte sich das Dogma ins Gegenteil verkehren, ohne dass dies in der Dritte-Welt-Bewegung auf Widerstände gestossen wäre? Wie in dieser Arbeit aufgezeigt wurde, machte es die Figur des Kleinbauern möglich, dass das ursprüngliche Konzept auf den Kopf gestellt wurde und eine paradoxe Situation entstand, die von der Dritte-WeltBewegung mitgetragen wurde.

In der Schweiz nahm die Umweltbewegung im Vergleich mit Grossbritannien oder der Niederlande sehr früh und sehr stark Einfluss auf die Dritte-Welt-Bewegung. Die beiden Lager können teilweise gar nicht auseinanderdividiert werden. Zudem setzte die Umweltbewegung Mitte der 1970er-Jahre ein, als der Impuls, der durch die „Erklärung von Bern“ entstanden war, bereits wieder an Stärke eingebüsst hatte. Die Umweltbewegung hat sich die Dritte-Welt-Bewegung einverleibt und mit dem Primat des Umweltschutzes deren Grundfesten erschüttert und deren Stossrichtung geändert. Sie hat die eigentlichen Wertvorstellungen der Dritte-Welt-Bewegung, die sie bis heute prägt, in eine andere Richtung gelenkt.

Die westlichen Staaten haben in den 1970er-Jahren und in der Folgezeit generell neoprotektionistische Gesetze eingeführt und insbesondere einen massiven Agrarprotektionismus aufgebaut. Gleichzeitig zur handelspolitischen Ausgrenzung erfolgte eine massive Erhöhung der Entwicklungshilfe für die ärmsten Länder. Die westlichen Gruppierungen, die 1968 noch anwaltschaftlich in den

Ә OpenAccess. (C) 2020 Andrea Franc, publiziert von De Gruyter. (cc) BY-NC-ND Dieses Werk ist lizenziert unter der Creative Commons Attribution-NonCommercial-NoDerivatives 4.0. 
Industrieländern für die Dritte Welt eingetreten waren, setzten sich schon bald für die biologische Landwirtschaft in ihren eigenen Staaten ein. Das Bild der Kleinbauernkooperativen, die in Dritte-Welt-Ländern als politische Akteure und Gegner rechter Militärdiktaturen auftraten, vereinfachte diesen Übergang diskursiv und trug ihn mit. Gleichzeitig reduzierten die Vertreter eines gerechten Handels ihren Einsatz, den sie für die Dritte Welt leisteten, auf den Kleinbauern und forderten für diesen im Rahmen des Gütesiegelsystems eine Verknüpfung aus genereller Entwicklungshilfe und besserer Bezahlung für dessen tropische Nahrungsrohstoffe. Diese Kombination, die von Organisationen wie der EvB gefordert und gefördert wurde, beinhaltete also die öffentliche, das heisst staatliche, von NGOs kritisch observierte Entwicklungshilfe sowie einen freiwilligen Beitrag der westlichen Konsumentinnen und Konsumenten beim Kauf eines tropischen Produkts. Der Fokus auf den Kleinbauern hatte beabsichtigte und unbeabsichtigte Folgen. Ursprünglich auch politisch motiviert, bereitete die Figur des Kleinbauern aber auch den Weg für die Kooperation mit der Umweltbewegung und liess den fairen Handel für die eigene Bevölkerung weniger systembedrohend erscheinen.

Indem der Blickwinkel auf die Figur des Kleinbauern reduziert wurde, geriet die Diskriminierung sämtlicher anderer Bevölkerungsschichten in Entwicklungsländern durch den westlichen Neoprotektionismus in Vergessenheit. Die Ausgrenzung der ärmsten Entwicklungsländer aus dem westlich dominierten Welthandelssystem wurde für die Dritte-Welt-Bewegung und ihre Anhängerinnen und Anhänger unsichtbar. Doch auch der Kleinbauer in Entwicklungsländern wurde durch den westlichen Agrarprotektionismus geschädigt. Er konnte seine nicht tropischen Produkte nicht mehr in den Westen verkaufen und seine Wettbewerbsfähigkeit wurde durch subventionierte Importe aus dem Westen untergraben.

Im Falle der Handelsbarrieren gegenüber nicht tropischen Produkten aus der Produktion des Kleinbauern wurde das Problem von Seiten der Dritte-Welt-Organisationen nie benannt. Es existierte gar nicht als Problem, denn das Primat des Umweltschutzes setzte die lokale Produktion voraus. Im Gegenteil: DritteWelt-Organisationen setzten sich trotz des Primats der regionalen und saisonalen Produktion für Importe tropischer Produkte aus kleinbäuerlicher Produktion ein. Aus Sicht der Bewegung hob man sich also durchaus von der Bio-Bewegung ab, indem man sich immerhin für den Transport und den Import gewisser Produkte aus dem Süden stark machte.

In den 1960er-Jahren nahmen die westlichen Entwicklungs-NGOs die Forderungen der UNCTAD auf und portierten diese. In meiner Doktorarbeit habe ich aufgezeigt, dass bereits im 19. und in der ersten Hälfte des 20. Jahrhunderts christliche Bewegungen versuchten, in Europa Absatzmärkte für die Produkte aus 
ihren Missionsfeldern zu schaffen. ${ }^{1}$ Im Licht dieser Beobachtung war der Einsatz von Dritte-Welt-NGOs für die Anliegen der UNCTAD in den 1960er-Jahren keine Zäsur, wie man sie von den Bewegungen dieser Zeit hätte erwarten können. Wie in dieser Arbeit dargelegt wurde, fand die überraschendere und für uns interessantere Entwicklung in den 1970er-Jahren statt. In dieser Zeit brachte die DritteWelt-Bewegung ihre Konsum- und Wachstumskritik vor. In dieser Arbeit wird aufgezeigt, wie gewisse Anliegen der Umweltschutzbewegung innerhalb der Dritte-Welt-Bewegung entstanden und so eine spätere, grossflächige thematische Überlappung von Dritte-Welt- und Umweltbewegung vorwegnahmen. Mit der Übernahme der Konsum- und Wachstumskritik kam die Dritte-Welt-Bewegung von den Forderungen der ersten UNCTAD im Jahr 1964 und von der wirtschaftswissenschaftlichen Grundlage ab. Insbesondere wandelte sich die Haltung der Bewegung gegenüber privaten Direktinvestitionen westlicher Firmen in Entwicklungsländer. Hatten die frühen Aktivisten in den 1960er-Jahren noch mehr private Direktinvestitionen im globalen Süden gefordert und die Ausgestaltung der Rahmenbedingungen diskutiert, so änderte sich diese Haltung in der ersten Hälfte der 1970er-Jahre in eine pauschale Ablehnung multinationaler Firmen und deren Aktivitäten in Entwicklungsländern. Die Bewegung begann, ein paraakademisches Konzept von Fair Trade $\mathrm{zu}$ entwickeln und $\mathrm{zu}$ propagieren, das immer weniger auf wirtschaftswissenschaftlichen Grundlagen basierte. Die Wegbereiter des Fair Trade haben der Makroökonomie ein Verständnis von Kultur bzw. Entwicklung gegenübergestellt, welches das globale Wohlstandsgefälle mit einer Pluralität der Werte erklärte und somit akzeptierte.

Diese Arbeit hat aufgezeigt, wie Dritte-Welt-NGOs den Neoprotektionismus ignoriert und in gewissen Fällen sogar aktiv gefordert haben. Die „unheilige Allianz“ zwischen der Dritte-Welt-Bewegung und der nationalkonservativen Rechten zieht sich seit Mitte der 1970er-Jahre wie ein roter Faden entlang wichtiger Eckpfeiler der Schweizer Geschichte. In den 1980er-Jahren waren die Dritte-WeltNGOs bei einem Konzept angelangt, das weder die Modernisierung der Entwicklungsländer noch den Marktzugang für deren nicht tropische Produkte vorsah. An die Stelle abstrakter ökonomischer Modelle war das Idealbild des Kleinbauern getreten. Erst dieses neue und fundamental andere Konzept legte die Basis für den Fair Trade, den wir zu Beginn des 21. Jahrhunderts kennen. Zu Beginn der 1980erJahre begann die Dritte-Welt-Bewegung, die sehr komplexe, akademisch fundierte Handelstheorie und die Implikationen der 1949 eingebrachten Prebisch-SingerThese auf ein kleines Symbol zu reduzieren. Die Vorgeschichte dieses Symbols bzw. die vorgängige Wandlung der Idee der Nord-Süd-Gerechtigkeit weist darauf

1 A. Franc: Wie die Schweiz zur Schokolade kam. 
hin, dass das heutige Konzept der Zertifizierung weiterhin gesellschaftlich debattiert werden sollte.

Die Tatsache, dass sich das Fair-Trade-Konzept so stark verändert hat, während sich die Dritte-Welt-Bewegung professionalisierte, wirft wiederum die Frage auf, welchen Nutzen diese Konzeptänderung den unterdessen professionellen NGOs brachte. Der ursprüngliche Grundsatz, dass Entwicklungsländern nicht (nur) mit Hilfe, sondern durch Teilhabe an einem gerechten Welthandel geholfen werden soll, wurde im Prinzip stets beibehalten. NGOs wie die EvB, die sich nicht auf eigentliche Hilfe, sondern auf Information und Überwachung einer gerechten Weltwirtschaft spezialisiert hatten, waren gegenüber Entwicklungshilfe im Sinne von Almosen stets kritisch eingestellt. Alleine damit zeigten sie sich gegenüber ihren Mitgliedern als kritische Beobachter der Entwicklungspolitik. Ausgerechnet die Kritik an der Hilfe als Almosen und eine vage Erinnerung an den Slogan Trade, not Aid wurden zu Promotoren des Gütesiegels. 